\title{
Implementation of low-dose CT screening in two different health care systems: Mount Sinai Healthcare System and Phoenix VA Health Care System
}

Claudia I. Henschke ${ }^{1,2 *}$, David F. Yankelevitz ${ }^{1,2 *}$, Artit Jirapatnakul ${ }^{1,2 *}$, Rowena Yip ${ }^{1,2 *}$, Vivian Reccoppa ${ }^{1 *}$, Charlene Benjamin $^{1 *}$, Tserling Llamo ${ }^{1 *}$, Angel Williams ${ }^{1 *}$, Simon Liu ${ }^{1 *}$, Daniel Max ${ }^{1 *}$, Samuel M. Aguayo $^{2 * *}$, Providencia Morales ${ }^{2 * *}$, Brian J. Igel ${ }^{2 * *}$, Hamed Abbaszadegan ${ }^{2 * *}$, Peter A. Fredricks ${ }^{2 * *}$, Daniel P. Garcia ${ }^{2 * *}$, Paska A. Permana ${ }^{2 * *}$, Janet Fawcett ${ }^{2 * *}$, Samir Sultan $^{2 * *}$, Lorenza A. Murphy ${ }^{2 * *}$

${ }^{1}$ Mount Sinai Healthcare System, New York, NY, USA; ${ }^{2}$ Phoenix Veterans Affairs Health Care System, Phoenix, AZ, USA

Contributions: (I) Conception and design: CI Henschke, SM Aguayo; (II) Administrative support: CI Henschke, SM Aguayo; (III) Provision of study materials or patients: All authors; (IV) Collection and assembly of data: All authors; (V) Data analysis and interpretation: All authors; (VI) Manuscript writing: All authors: All authors; (VII) Final approval of manuscript: All authors.

Correspondence to: Claudia I. Henschke, PhD, MD. Icahn School of Medicine at Mount Sinai, One Gustave Levy Place, New York, NY 10029, USA. Email: Claudia.Henschke@mountsinai.org; Samuel M. Aguayo, MD. Phoenix VA Health Care System, 650 E Indian School Road, Phoenix, AZ 85012, USA. Email: Samuel.Aguayo@va.gov.

\begin{abstract}
Implementation of lung screening (LS) programs is challenging even among health care organizations that have the motivation, the resources, and more importantly, the goal of providing for lifesaving early detection, diagnosis, and treatment of lung cancer. We provide a case study of LS implementation in different healthcare systems, at the Mount Sinai Healthcare System (MSHS) in New York City, and at the Phoenix Veterans Affairs Health Care System (PVAHCS) in Phoenix, Arizona. This will illustrate the commonalities and differences of the LS implementation process in two very different health care systems in very different parts of the United States. Underlying the successful implementation of these LS programs was the use of a comprehensive management system, the Early Lung Cancer Action Program (ELCAP) Management System ${ }^{\mathrm{TM}}$. The collaboration between MSHS and PVAHCS over the past decade led to the ELCAP Management System ${ }^{\mathrm{TM}}$ being gifted by the Early Diagnosis and Treatment Research Foundation to the PVAHCS, to develop a "VA-ELCAP" version. While there remain challenges and opportunities to continue improving LS and its implementation, there is an increasing realization that most patients who are diagnosed with lung cancer as a result of annual LS can be cured, and that of all the possible risks associated with LS, the greater risk of all is for heavy cigarette smokers not to be screened. We identified 10 critical components in implementing a LS program. We provided the details of each of these components for the two healthcare systems. Most importantly, is that continual re-evaluation of the screening program is needed based on the ongoing quality assurance program and database of the actual screenings. At minimum, there should be an annual review and updating. As early diagnosis of lung cancer must be followed by optimal treatment to be effective, treatment advances for small, early lung cancers diagnosed as a result of screening also need to be assessed and incorporated into the entire screening and treatment program.
\end{abstract}

Keywords: Screening; Veterans Administration; health care systems; computed tomography

Submitted Jun 19, 2020. Accepted for publication Nov 10, 2020.

doi: $10.21037 /$ tlcr-20-761

View this article at: http://dx.doi.org/10.21037/tlcr-20-761

*, for the Mount Sinai Healthcare System.

**, for the Phoenix VA Health Care System. 


\section{Introduction}

Most patients who have been diagnosed with lung cancer while participating in lung screening (LS) programs have been cured (1-4), in contrast to the tragic prognosis of patients with lung cancers that are incidentally discovered because of symptoms or abnormal clinical tests such as imaging or laboratories $(5,6)$. And yet, implementation of LS programs remains a challenge even among health care organizations that have the motivation, the resources, and more importantly, a great number of people who are at high risk of suffering from lung cancer or from co-morbidities that can also be detected with the same low-dose CT (LDCT) screening of the chest (7). These comorbidities include coronary artery and aortic valve disease and pulmonary obstructive disease which account for more deaths than lung cancer each year as well as other diseases of the organs in the chest and upper abdomen.

The introduction of helical CT scanners in the early 1990's made CT screening for lung cancer realistic as for the first time the entire chest could be scanned in a single breath-hold that avoids missing parts of the lungs (1-4). The Early Lung Cancer Action Project (ELCAP) was the first cohort study of 1000 high-risk smokers which started in 1992, and it showed that with LDCT screening a high proportion of the cancer diagnoses occurred in Stage I which had high survival rates (2-6). It also provided the initial management protocol $(2,3)$ and later the long-term survival rates (4). Similar results were reported in the Nagano region population study, which started in Japan in 1996 under the direction of Professor Sone and his colleagues (8). Subsequently, two large scale randomized trials, the National Lung Screening Trial (9) and the DutchBelgian Lung Cancer Screening Trial (NELSON) (10) confirmed the clinical benefit of LDCT screening for lung cancer. In 2014, the United States Preventative Task Force (USPSTF) gave a "B" rating to LDCT screening (11) which meant that medical insurance companies were required to cover the cost of the LDCT screening. It also led to the decision by Centers for Medicare and Medicaid Services (CMS) to reimburse LDCT screening starting in 2015 (12). According to CMS, LS is indicated for asymptomatic persons who are free of potential manifestations of lung cancer which include worsening cough with hoarseness or hemoptysis, and unexplained weight loss. If on the other hand these symptoms are present, clinical workup of their symptoms should be provided.

It is estimated that more than 222,500 new cases of lung cancer will be diagnosed in 2020 in the United States, including about 44,500 in never smokers (13). Meanwhile, the current CMS guidelines for LS (age 55 to 77, 30 packyears of smoking or more, quit within the last 15 years) already appear obsolete since only about $20 \%$ of the people diagnosed with lung cancer each year in the United States met the CMS criteria (14-16). Furthermore, even after the approval for LS reimbursement by CMS in 2015, national implementation of LS programs is estimated to have only enrolled about $4 \%$ to $15 \%$ of all eligible smokers by the current CMS guidelines (17-20). Hence, there is a pressing need for screening more people at risk, which in turn will provide more data for continuously improving the guidelines for LS.

The low rate of screening enrollment is in part due to the complexity and time to implement the required LS processes and quality assurance elements. In any given setting, it requires close coordination between many different healthcare professionals, infrastructure and support from stakeholders, including community outreach specialists, dedicated LS coordinators, patient and lung cancer advocacy groups, an integrated multidisciplinary team, nurse practitioners, epidemiologists, computer and data scientists, administration, billing, and compliance with regulatory agencies. It takes a dedicated team to implement a successful LS program.

Mount Sinai Healthcare System (MSHS) is a complex not for profit health care organization that is structured around eight hospital campuses and the Icahn School of Medicine at Mount Sinai. The eight hospitals are: Mount Sinai Beth Israel, Mount Sinai Brooklyn, Mount Sinai Hospital, Mount Sinai Queens, Mount Sinai Morningside (formerly Mount Sinai St. Luke's), Mount Sinai West (formerly Mount Sinai Roosevelt), New York Eye and Ear Infirmary of Mount Sinai, and Mount Sinai South Nassau. MSHS includes more than 6,600 primary and specialty care physicians and 13 ambulatory surgical centers, serving one of the most diverse populations in the U.S. It has ambulatory practices throughout the five boroughs of New York City, Westchester County, and Long Island, along with more than 30 affiliated community health centers. During the 2017-2018 fiscal year, it employed more than 39,000 people and reported 136,528 inpatient admissions, and 500,901 Emergency Department visits. The Icahn School of Medicine at Mount Sinai had 33 multidisciplinary research, educational, and clinical institutes. Since 2010, the MSHS LS program has been steadily increased to over 4,000 screenings per year. 
The Phoenix Veterans Affairs Health Care System (PVAHCS) is a federally funded health care organization, one of the busier VA facilities in the United States, serving almost 100,000 Veterans of which at least 24,000 are known or suspected of being smokers at some time in their life. The PVAHCS is also the main tertiary level care facility for the Northern Arizona VA Health Care System (NAVAHCS) in Prescott, Arizona, that serves another 28,000 Veterans including more than 5,000 smokers at risk. During calendar years 2015 and 2016, before full implementation of our current LS program, the PVAHCS provided care for 606 Veterans with a diagnosis of lung cancer, about half of them diagnosed at the PVAHCS and the others diagnosed elsewhere, with the most frequent age at the time of diagnosis being between 65 and 69 years old, and 28 (4.6\%) Veterans diagnosed before the age of 55. More than half of those Veterans with lung cancer died less than a year from a late stage diagnosis, and none of the 606 Veterans were diagnosed with lung cancer while participating in our earlier LS efforts (21).

Since our current LS program was supported by our PVAHCS leadership in 2018, we have evaluated more than 2,300 Veteran's electronic medical records (EMRs), interviewed and conducted shared decision making (SDM) with almost 1,100 Veterans (the others were not contacted because of not meeting eligibility criteria upon review of the EMR), enrolled 678 Veterans in our annual LS program (the others not meeting eligibility criteria upon telephone conversations, or declining to participate at the time of the interview, or diagnosed already with possible lung cancer). Of the enrolled Veterans, 480 have already had at least the baseline screening LDCT (the others already scheduled for LDCT at a date and time of their preference). Several early stage lung cancers have already been identified by LS and treated, suggesting that a possible cultural shift is already occurring with the realization that the best opportunity for saving Veterans lives from lung cancer is through LS for earlier detection and treatment, and that perhaps we are on our way for a significant downstaging of lung cancers diagnosed at the PVAHCS.

The challenges and opportunities for implementing an effective LS program at each of these very different health care organizations have both similarities and differences. Working together and learning from each other, staff from MSHS and PVAHCS have developed and described strategies, procedures, and tools for implementing LS programs that can be adopted or further adapted elsewhere to better serve people at risk. An example of this collaborative benefit is the ELCAP Management System $^{\mathrm{TM}}$, gifted by the Early Diagnosis and Treatment Research Foundation to the PVAHCS, for additional development and adaptation as a "VA-ELCAP" version of the original ELCAP Management System ${ }^{\mathrm{TM}}$. Today, an even more comprehensive translation of the "VA-ELCAP" management system into VA computer MUMPS language is already happening with support from a generous grant from the Bristol Myers Squibb Foundation. When completed, the PVAHCS will serve as the lead institution for the implementation and clinical testing of the system before possible dissemination to all other VA institutions (22).

MSHS had participated in the New York (NY)-ELCAP study with other institutions in New York State (23) and the International (I)-ELCAP study $(4,24)$, both of which started in 2001. In 2010, the ELCAP investigators brought the screening program they had started in 1992 at Weill Cornell Medical Center to MSHS. At MSHS, screening was performed under an IRB-HIPAA compliant research protocol to smokers and never-smokers, aged 40 and older. In 2015, a CMS-approved LS program was added for people who met CMS eligibility criteria. Of note, although the clinical investigators had been at the forefront of screening since the early 1990's (1-4), the implementation of a CMS authorized program throughout the MSHS still proved to be a daunting effort.

At the PVAHCS, LS efforts began in 2012 when authorization was obtained for a small quality improvement demonstration project for 1,000 Veterans. The VA had long recognized that lung cancer is the most common cause of cancer death among both men and women Veterans, and that the VA serves more smokers at high risk than other health care systems, in part because of earlier policies about smoking in both the military and the federal workplace (25). In 2012, a small VA Collaborative System Redesign Project on Lung Cancer had succeeded in reducing the time it took from suspected lung cancer from incident imaging to a confirmed histopathologic diagnosis, and then to appropriate treatment, but the vast majority of lung cancers diagnosed in the VA were still not in early stage and therefore poor clinical outcomes remained largely unchanged. It was intuitively clear that to reduce deaths from lung cancer and other smoking-associated co-morbidities like chronic obstructive pulmonary disease (COPD) and coronary arteries disease (CAD), the VA need to detect these at a much earlier stage, and for this reason the PVAHCS adopted the already published ELCAP protocol which in addition to lung cancer also focused on evaluation of CAD 
and COPD using the same LDCT scan (24).

That local PVAHCS decision was not without controversy because in 2012 the VA had not yet developed a national policy for LS, like most other health care organizations and third party payers, and because there were valid concerns about the possibility of overwhelming our limited health care manpower and technologic resources, both on the forefront end in primary care, as well as in radiology and pulmonary medicine. In addition, lung cancer stigma was another barrier to LS with many of the Veterans whom we invited to participate struggling with feelings of guilt for having smoked, or for not being able to quit, sometimes verbalizing feelings of diminished self-worth and hesitation about consuming LS health care resources that in their opinion could better serve others that were perhaps more deserving. The timid ways in which community advocacy partners and sometimes even academia slow walked towards embracing LS, and the spreading of the message that LS can save lives, did not help our Veterans receive better information or gain a better understanding of the potential benefits of LS.

Since 2012, staff from PVAHCS began to regularly participate and present at the semi-annual screening conferences held by the International ELCAP (I-ELCAP) investigators (26). The specific topic of how to better serve our Veterans by implementing the I-ELCAP screening protocol in the VA became a special emphasis section of these conferences, where attendees from other programs and from other countries began to learn about the VA, and some began to implement a few of our PVAHCS strategies given the specific circumstances in their countries. Staff from PVAHCS also adopted some strategies and procedures from MSHS, and then further adapted what needed to change based on the unique circumstances of the PVAHCS.

In LS, not unlike the experience of implementing breast cancer screening with mammography, there are complex evidence-based clinical algorithms that must be followed when interpreting images to avoid the risks of unnecessary diagnostic procedures. Despite a limited budget, a short study period, and a study design that relied on medical records review for evaluating outcomes of baseline CT screening, an early LS pilot study at eight VA facilities between 2013 and 2015 (27) provided enough data to depict challenges and opportunities for successful implementation of LS in the VA. Among other observations, the VA study reported considerable variability among the eight sites, demonstrating almost 3 -fold difference or over $50 \%$ variability in abnormal findings between sites (31-85\%), which was more than 3-times than is commonly reported in the literature. The variability should be approximately $10 \%$ as has been demonstrated in other screening programs in the US (28-30). That variability among those 8 VA facilities highlighted the need for a standardized protocol to evaluate, report, and then manage CT findings. The eight VA facilities also showed considerable variability in Veterans agreeing to be screened, ranging between 37\% and $65 \%$. This highlighted the need to improve the shared decision making (SDM) processes used to invite patients for screening, particularly for those with poor health literacy who may misinterpret the information, or who may feel overwhelmed by the effort to understand complicated ways of explaining the risk to benefit ratio. Veterans who live in rural areas or who have challenging circumstances for transportation may also benefit from better personalized outreach and intervention processes. Less emphasized, but in our opinion of greater significance, 20 of the 31 (65\%) lung cancers diagnosed with the initial CT screening were in Stage I, encouraging results that were never reported in the VA.

This variability in the implementation of LS programs had been recognized by the ELCAP investigators (24,31). The centralized ELCAP Management System ${ }^{\mathrm{TM}}$ allowed for ongoing quality assurance reviews by expert chest radiologists at the central I-ELCAP office to ensure appropriate image acquisition, interpretation, and coding. The reviews provided discrepancy reports with conference calls to discuss the summary findings. A dual reading and quality assurance (QA) process, used among all I-ELCAP screening institutions, found that new LS programs are more likely to report positive findings when compared to the formal interpretation at the I-ELCAP center, perhaps because of the concern about missing a lung cancer. The process was improved through training, dual readings, and successive evaluations through continuous learning (31).

This I-ELCAP QA system was adopted at the PVAHCS through a similar but intra-mural system with MSHS I-ELCAP radiologists joining the medical staff at PVAHCS, and after reviewing the first 400 baseline LDCTs, we found that only $14 \%$ required additional diagnostic testing, most of them a short-term follow up LDCT. In collaboration with the VA National Teleradiology Program, PVAHCS is currently exploring the potential for expansion of this QA service to any VA facility that is offering LS, like how it is offered to all other I-ELCAP programs worldwide.

The success of the initial ELCAP screening program that started in $1992(1,2)$, and subsequently of all its 
successor projects, the NY-ELCAP (23) and the current I-ELCAP (24), was facilitated by the ELCAP Management System $^{\mathrm{TM}}$ software for management and QA, allowing for storage of both radiologic images and clinical data (32). The use of the I-ELCAP protocol and the ELCAP Management System ${ }^{\mathrm{TM}}$ software functions as a digital platform for the emerging data to be pooled and analyzed in an efficient manner. It provides for worldwide QA, allows for continual review of screenings, and to date has resulted in over 300 publications that have shaped the approach to LDCT screening. Since the earliest days of I-ELCAP, and through each of its conferences (26) and evolution of the ELCAP Management System ${ }^{\mathrm{TM}}$ software, there has been an appreciation for the power of collaboration and open science. This same philosophical underpinning inspired the distribution of the ELCAP Management System ${ }^{\mathrm{TM}}$ software to more than 82 healthcare institutions in 10 countries, and also to the PVAHCS through a generous no cost license from the Early Diagnosis and Treatment Research Foundation in New York. The licensing authorizes further VA specific development of the software and then use of the "VA-ELCAP" version of the software to efficiently and safely enroll in LS the thousands of Veterans at risk that we serve at the PVAHCS. The license also authorizes and facilitates the use of the accumulating LS data for QA, for training, and for collaborative research. To further enhance the "VA-ELCAP" version of the ELCAP Management System $^{\mathrm{TM}}$ software, to fully integrate the software with the Veterans Health Information Systems and Technology Architecture-Computerized Patient Record System (Vista-CPRS), that is the VA's electronic medical records (EMR) system, and to eventually make the "VA-ELCAP Management System" software available to all other VA facilities, there is an ongoing effort to translate the original ELCAP Management System ${ }^{\text {TM }}$ software into computer MUMPS language, the specific code used for the current VA Vista-CPRS EMR system $(22,33)$.

\section{Critical components of a LS program and solutions for implementation}

In future reports, we will describe the challenges and the technical aspects of developing the ELCAP Management System $^{\mathrm{TM}}$ software that is specific for the VA. Here we will address the non-software challenges in each of our health care systems, and what we consider are the critical components in implementing a LS program, including:

(I) Current indications for screening and the need for re-evaluation of criteria;

(II) Meeting the requirements for authorized screening programs;

(III) Selecting the screening regimen and protocol;

(IV) Identifying and contacting people eligible for screening and educating physicians;

(V) Providing information of the benefits and risks of screening to a person prior to screening;

(VI) Providing smoking cessation information and options;

(VII) Obtaining the LDCT and calibration of the CT scanner and protocol performance;

(VIII) Interpretation of the LDCT results and providing follow-up recommendations;

(IX) Screening management system;

(X) Quality assurance, feedback, and outcomes analyses.

The details of each of these components need to be specified and then continually re-evaluated by an ongoing QA program and the database created by the actual screenings. There should be at least annual reviews of the entire process and updating as needed based on ongoing review of the data.

Updating is needed because advances in CT scanner technology have been remarkable and are expected to continue. More powerful hardware and image reconstruction algorithms have allowed for faster scanning at lower radiation doses in today's multi-detector CT (MDCT) scanners that is reaching the dose of a chest radiograph. The thinner collimation of modern CT scanners has led to the detection of smaller pulmonary nodules. Along with advances in CT imaging, diagnostic techniques have rapidly developed such as percutaneous biopsies, navigational bronchoscopy, and PET scans. These advances have altered clinical care and they need to be integrated into the ongoing screening program to maximize the benefit and minimize risks of screening. Treatment advances have also been made.

\section{Current indications for screening and the need for re- evaluation of criteria}

The current CMS indications are primarily based on the enrollment criteria of the NLST, hence, CMS reimbursement for LDCT screening is restricted to asymptomatic smokers aged 55 to 77 who have a 30-packyear history of smoking and are currently smoking or quit within the last 15 years (12). The National Comprehensive 
Cancer Network (NCCN) indications are for asymptomatic current or former smokers who are, Group 1: same as the CMS criteria or Group 2: aged 50 years and older with at least 20 pack-years smoking history and at least one other risk factor among the following ones (personal history of smokingrelated cancer, family history of lung cancer in first-degree relatives, occupational exposure to known lung carcinogens, radon exposure or chronic lung disease including emphysema and pulmonary fibrosis) (34). Multiple efforts are ongoing to expand the criteria for screening because some $80 \%$ of lung cancer patients diagnosed in the United States still fall outside the current CMS criteria. While LS is restricted to asymptomatic people, other co-morbidities like COPD and CAD have similar symptoms of cough, dyspnea, and chest pain. Thus, good clinical judgement is imperative.

The VA may prove the ideal clinical setting to continuously evaluate and better refine the proper indications for LS. For instance, lung cancer occurs in Veterans at younger age and with lower cumulative exposure to tobacco, raising the possibility that other unknown military exposures or familial effects may also need to be considered (35). The NLST, NCCN, and CMS criteria for LS do not address any of these considerations. Similarly, women appear to suffer from lung cancer at lower cumulative exposure to tobacco and then have worse outcomes, making them another special group to benefit from LS $(10,36)$. And while COPD is recognized as an independent important risk factor for lung cancer (37), none of the current screening criteria has matured to offer LS to smokers with COPD when they do not meet the current criteria, except for the NCCN criteria. The VA has more smokers at high risk than other health care systems, many Veterans also suffering from COPD, and more than $10 \%$ of Veterans are women. Thus, as the LS database expands in the VA, it will provide opportunities to further study and refine criteria for LS.

\section{Meeting the requirements for authorized screening programs}

In the United States, the minimum qualifications for having a screening program have been defined by CMS include that each CT scanner must be certified for screening by the American College of Radiology (ACR). At MSHS, accreditation by ACR, an application to ACR for each CT scanner used for LS throughout the MSHS, including details of the CT scanner and low-dose acquisition protocol.

CMS also requires that radiologists must have read a minimum number of $300 \mathrm{CT}$ scans in the past 3 years, a LS protocol used for interpretation of the LDCT and recommendations must be specified, and the data from the report must be submitted to CMS through an approved registry.

Although not required by CMS, but clearly stated in other international screening guidelines $(38,39)$, other elements for certification should include quality oversight of LDCT interpretations, a dedicated navigator to ensure timely transition from diagnosis to treatment, access to a thoracic oncology multidisciplinary team, and continuous real-time feedback of the short-term and long-term results of the screening program. As shown in Figure 1 (40), these elements which are all a component of the I-ELCAP protocol result in better clinical outcomes for the participants in the screening program.

The PVAHCS also participated in using the recommended Radiological Society of North America (RSNA) phantom for assessing the performance of the CT scanner used for LS $(41,42)$. In addition, the PVAHCS follows the VA internal memorandum of guidance (official correspondence from VA Deputy Under Secretary for Health for Operations and Management, November 2017) for providing LS to Veterans at risk, with many elements that are like those mentioned above for the ACR accreditation. PVAHCS meets every one of the VA guidance elements, with two notable exceptions that warrant further discussion.

First, because of our commitment to Veteran-centric preferences, the PVAHCS does not require a face to face visit to discuss LS with the Veteran at risk, avoiding burdensome logistics of time, space (parking, clinic office, waiting room), and scheduling before we even know if the Veteran is a candidate who could benefit from imaging with LDCT, or who is interested in participating in LS. Instead, we successfully execute a process that we have named "Precision Outreach", where we first evaluate the Veterans' EMR and then, if appropriate, talk to the Veteran on the phone to verify risk factors and the possibility of medical benefit from LS before inviting them to participate. SDM is also conducted over the phone and documented in the EMR. Only after the informed Veteran opts to participate in our LS program, an order is placed to schedule the LDCT. This telephone clinic for "Precision Outreach" enrollment of Veterans in LS was successful before the 


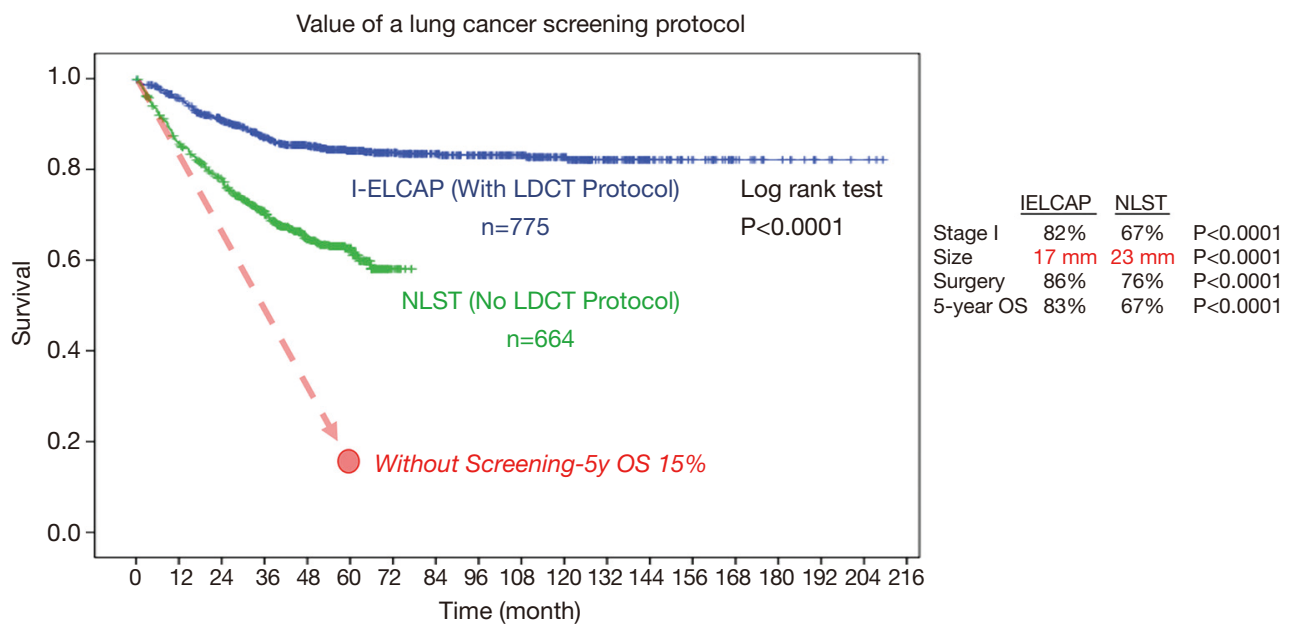

Figure 1 Lung screening program with a structured protocol is associated with further gains in survival than that demonstrated in the NLST. This comparison of lung cancer specific survival between the patients diagnosed with stage I NSCLC in the I-ELCAP and NLST protocols reveals the potential magnitude of benefit when a structured LS protocol is established to guide elements of LDCT acquisition, image interpretation, and a clinical management workflow that ensures the timely transfer of patients from diagnosis to treatment. [Adapted from Yip, Henschke, Yankelevitz et al., 2015 (40)]. LDCT, low-dose CT.

COVID-19 pandemic, and it did not require significant changes during the COVID-19 crisis. In fact, other clinical services are now emulating this Veteran-centric process in order to decrease non-essential face to face interactions with patients, an effort that is greatly appreciated by many of our Veterans. More details about this "Precision Outreach" process presented below.

The second deviation from the general VA guidance memorandum and most other VA facilities is that the PVAHCS adopted the I-ELCAP screening protocol (24) instead of the ACR LungRADS protocol (43), because in our own research the I-ELCAP protocol has been found to be just as safe and yet at least 30\% more efficient, that is requiring $30 \%$ fewer unnecessary workup and biopsy procedures for one lung cancer detected (44-46). As the VA serves about 1 million smokers who are potentially at risk and eligible for LS, this increased efficiency of the I-ELCAP protocol has significant implications both for patient safety and for allocation of resources.

\section{Selecting the screening regimen and protocol}

The importance of a carefully defined screening regimen was highlighted in the comparison of I-ELCAP results and NLST results (40). I-ELCAP used a defined algorithm, while the NLST specifically state that it did not specify one (9).
I-ELCAP had a higher frequency of Stage I lung cancer diagnoses $(82 \%$ vs. $62 \%, \mathrm{P}<0.0001)$ and a smaller median cancer size $(17$ vs. $23 \mathrm{~mm}, \mathrm{P}<0.0001)$.

The screening protocol specifies the entire process of the pursuit of early, rule-in diagnoses of lung cancer. It begins with the initial, baseline low-dose CT scan and continues with repeat screenings. It defines a positive result of each round of screening and the follow-up diagnostics. As the frequencies of different cell-types of lung cancer in the baseline round are different from those diagnosed in repeat rounds of screening, the definition of a positive result and the subsequent algorithm are defined separately for the baseline and subsequent annual repeat rounds (45). It is also understood that there may need to be occasional exceptions to the protocol based on clinical and imaging findings. When the algorithm is applied in each screening round and does not lead to the diagnosis of malignancy, the next repeat screening is scheduled at a preset time. It was also shown that some solid and many subsolid pulmonary nodules resolve spontaneously, particularly new ones identified on repeat screening examinations (47). More than $70 \%$ of new nodules identified on annual repeat screening studies resolved by the time of the 1-month follow-up CT. Thus, follow-up imaging three months after baseline or one month after annual repeat screening is useful to avoid unnecessary diagnostic interventions, especially invasive 
diagnostic interventions.

\section{Identifying and contacting the people eligible for screening and educating physicians}

Providing the information about the availability and benefits of LS to the eligible people and to their physicians is a challenging task. It requires a multi-faceted process which includes giving presentations in the community and physicians forums, reaching out to the relevant advocacy groups, religious institutions, and general advertising. In addition, the VA outreach efforts must include regular communications with Veterans Service Organizations (VSO) and VA leaders, as well as sharing the information with political representatives who have influence over service priorities and allocation of resources.

The public and physicians must believe that there is a benefit to the screening, and that the biggest risk of all may be to not screen people who are at high risk. The physicians also need to feel that the scheduling and follow-up of their referrals will be appropriate, and reassured that there is a clear delineation of who has the responsibility for followup of findings and also follow-up for continued annual screenings, in partnership with those patients who agree to participate in LS. It also needs to be clear that it is the annual rounds which provide the life-saving early diagnosis, and that the baseline LDCT is only the entrance to annual LS.

At MSHS, we gave talks at our medical centers in all the boroughs of New York City and surrounding areas. We placed information bulletins in local newspapers, local media, and kiosks. We worked with advocacy groups. We reached out to physicians, particularly the general medicine, pulmonologists, and thoracic surgeons. We assured physicians, that if they wanted, we would be responsible for screening enrollment and follow-up process. We would keep them informed about all the steps including obtaining prescriptions for the screenings. This included determination of eligibility, scheduling of screenings, and follow-up. Pop-up protocols were installed in our EMR to alert the practitioner to discuss the availability of the screening during the medical visit, depending on the smoking history and age. Some of the MSHS medical centers wanted to use this approach while others did not as they already received an overwhelming number of clinical alerts. We streamlined the LS and SDM ordering process so that the physicians' orders are completed more expeditiously and accurately. Most physicians are very supportive of the LS program but are concerned about any new process that might take time away from their patients. We anticipated that, included them in the conversation, and met their needs. Educating the physicians and other clinicians (nurse practitioners and physician assistants) was critical to the success of the LS program. We emphasized that one of the hallmarks of the LS program was that we did not add to their workload, but in fact made caring for their patients with smoking history easier, and that we were flexible enough to adapt to changing demands. Working closely with the EPIC EMR coders was labor intensive but well worth the effort as many small changes were required due to multiple systems within MSHS.

Implementation of LS at the PVAHCS faced many of the challenges described above for MSHS, and we also communicated with primary care providers (PCP) to assure them that our LS program would help them provide better care as a team, without adding more time requirements to their clinics. To keep that commitment, our LS staff conducts a thorough review of the Veteran's EMR, evaluating documented risk factors and any obvious reasons that would negate potential medical benefits of LS, for example the presence of signs or symptoms suggestive of lung cancer, another debilitating terminal disease, advanced old age, or mental health conditions rendering the patient not competent for understanding and consenting to participate in LS. Then, if appropriate, we contact the Veteran at the preferred telephone number during working hours, to verify demographics, the presence of risk factors, and the potential for medical benefit from LS. During this telephone conversation with the Veteran, we use the EMR as a reference but also taking the opportunity to make the EMR more complete and more accurate using our LS program note template. For example, we not only verify that the Veteran is in fact a cigarette smoker but also quantify the cumulative smoking history in packs per years and document it as a retrievable variable that can be used whenever searching EMRs for patients at risk. Sometimes we document that the Veteran is a smoker, but that the smoking habit is only the occasional use of pipe, cigars, or marijuana, none of which would require annual LS based on current guidelines. Other times we document that the Veteran used to smoke cigarettes, but without a significant cumulative exposure because they quit many decades ago, or that in spite of having all risk factors and meeting eligibility criteria, the Veteran is not interested in participating in LS, or at least not ready to decide at the time of the communication. If the Veteran is a current smoker, we offer smoking cessation information and a referral to our 
smoking cessation program, sometimes alerting the PCP about the Veteran's interest in smoking cessation resources. Finally, if the Veteran is eligible and voices interest in LS, we discuss the latest available information about potential benefits and risks of LS, documenting SDM in the EMR, and if the Veteran opts to participate, we also place the order for scheduling an LDCT at the convenience of the Veteran. In this way the only time when the Veteran needs to travel is when the LDCT is to be performed, and by the time the Veteran presents for the LDCT, we all know it is the right person, for the right test, at the right time, with minimal waste of time, effort, and resources by PCPs, radiologists, and other VA staff. All that is required for this process to be initiated is a referral of the Veteran to our LS program, usually via electronic consultation (eConsult), or an alert notice from radiology to our LS program staff about an LDCT performed on one of our Veterans.

The process described above was well received at the PVAHCS, supported by clinical leaders in radiology, pulmonary, and primary care, and approved by our Clinical Executive Board and facility leaders. But the low numbers of Veterans enrolling in LS did not match our expectations based on the number of Veterans at risk. Providing a great service for many Veterans at risk and who could benefit but enrolling only a few in LS did not make sense. Our LS staff concluded that this process was too passive and depending of other busy clinicians to "push" their Veterans toward LS. Thus, our strategy changed, and we turned the process into a more active "pull" system where our LS staff began to contact Veterans at risk instead of waiting for eConsults and other referrals. To accomplish this, the key task became how to identify our Veterans at risk, and for that our Clinical Informatics staff developed a tool, named the Phoenix ELCAP Dashboard, leveraging actionable data that already existed in the EMR database.

The Phoenix ELCAP Dashboard is a straightforward data retrieval of tobacco related patient indicators from patient medical screening questionnaires and/or tobacco International Classification of Disease-10 (ICD-10) diagnoses completed over the last 5 years. In the VA, like in many other health care systems, patients are routinely surveyed about their tobacco usage. Health screening results are entered directly into the EMR by qualified medical providers and risk-stratified based on their survey responses. Smoking histories are assigned broad categories such as "quit tobacco $>7$ years ago" or "current tobacco user," but unfortunately there have not been any specific health factors quantifying cumulative smoking history in pack per years, that could facilitate the listing of Veterans at high risk based on current LS criteria. Therefore, LS staff adapted the LS intake form of the VA-ELCAP Management System to create a facsimile note template in the Vista-CPRS EMR, to facilitate the transfer of the needed information. The VA corporate data warehouse (CDW) compiles almost all aspects of the EMR, including results of these medical questionnaires and all ICD-10 coding. All that was needed was to retrieve the potential cohort of Veterans at risk from the CDW. Candidates with tobacco use indicator were listed on the dashboard that was created using Structured Query Language (SQL) data retrieval. Only the most recent tobacco positive survey response or ICD-10 code was displayed, and only for Veterans alive at the time of the query. Veterans and their findings would be represented on the dashboard if they had either a positive survey finding of tobacco use, or a tobacco related ICD-10 code, or both. The original Phoenix ELCAP Dashboard also shows whether the Veteran has a diagnosis of COPD and the remoteness (rurality) of their home of record to one of our VA facilities, as defined by the VA Office of Rural Health (ORH) in Washington, DC. Another feature of the dashboard for case finding by the LS staff is the display of the most recent LDCT or chest/thoracic CT result for each subject where available. The Phoenix ELCAP Dashboard template can be further modified for other variables of interest, and then installed and used at any VA facility. Using this "precision outreach" cohort from the Phoenix ELCAP Dashboard, the LS staff at PVAHCS can act deliberately to contact and promote LS to individual Veterans who, after a thorough review of their EMR, are suspected to be at high risk and may benefit from LS.

Since the development of the Phoenix ELCAP Dashboard in 2017, LS staff at the PVAHCS have implemented three access pathways for Veterans to participate in LS: Precision Outreach, eConsults from PCP or other clinicians, or direct notices from Radiology after a Veteran had an LDCT ordered by any clinician (Figure 2). The eConsult pathway is a mechanism for direct referral of potential high-risk Veterans from ambulatory clinics and post hospital discharge, and it is designed for the concerned clinician who would like to offer this service to the Veteran but does not have enough time for appropriate SDM and documentation, or who prefers to ensure continuous follow up of the Veteran by our LS staff. Direct notices from Radiology are automatically generated from the EMR of any Veteran who receives an LDCT, regardless of what clinician ordered the LDCT. This notice prompts LS staff 


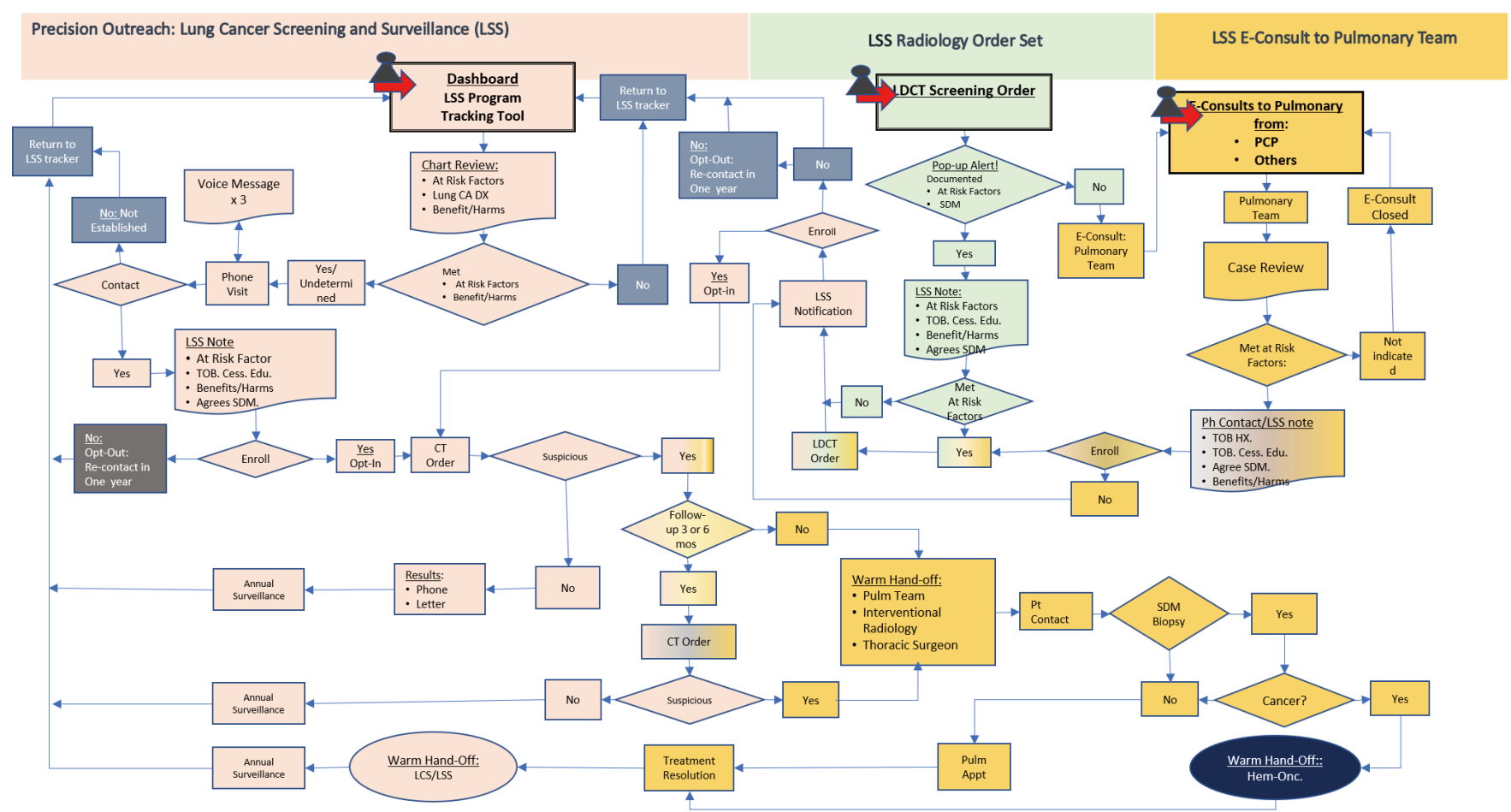

Figure 2 Pathways to enroll Veterans in the LS program at PVAHCS. Up to date, eConsults comprise $14 \%$ of our total LS enrollment and another $1 \%$ are direct notices from Radiology after an LDCT is performed on a Veteran. The main pathway for participation is through Precision Outreach, contributing $85 \%$ of the total LS program enrollment. Please note that the three different pathways for enrollment are part of a "closed loop" system to ensure that no Veteran falls out of sight from our LS staff. LDCT, low-dose CT.

to review the Veterans EMR, determine whether the LDCT was ordered for screening and whether it was appropriate, sometimes creating an opportunity for more education of clinicians and for creating new partnerships to support LS for Veterans. Nevertheless, Precision Outreach has become the main pathway for enrolling Veterans in LS at PVAHCS, responsible for $85 \%$ of our enrollment. Since all PCP and other providers are fully aware of the program and welcome the service to our Veterans, there has been a cultural shift in the appreciation for LS and the diagnosis of lung cancers at an early stage.

\section{Providing information of the benefits and risks of screening to a person prior to screening}

The full benefit of screening becomes manifest only when screening continues over many rounds of annual screening. The mortality reduction from lung cancer achieved by continued screening is higher than the $20 \%$ mortality reduction reported for the NLST (9). The NLST, correctly stated, showed a $20 \%$ mortality reduction of LDCT over chest radiographic screening after 3 rounds of screening and an average 5 years of follow-up without screening. If screening had continued in the NLST, the mortality reduction would have been greater; some estimate close to $70 \%$ or more. As such a trial would cost more than 1 billion dollars and would had taken over a decade to complete, a shorter trial design was used for the NLST. The trial was designed to detect a minimum mortality reduction of $20 \%$ in the CT arm. If this reduction could be achieved, the trial would be stopped and it would be declared that LDCT screening was beneficial.

LDCT screening detects smaller cancers than in the absence of screening $(5,48-50)$. Using the large SEER database, it has been shown that estimated cure rates decrease for each $1 \mathrm{~mm}$ increase in tumor size. The $8^{\text {th }}$ edition of the American Joint Committee on Cancer (AJCC) staging (51) identified that the survival probability of NSCLC progressively declines with every millimeter of tumor growth that can result from delays in initiating 


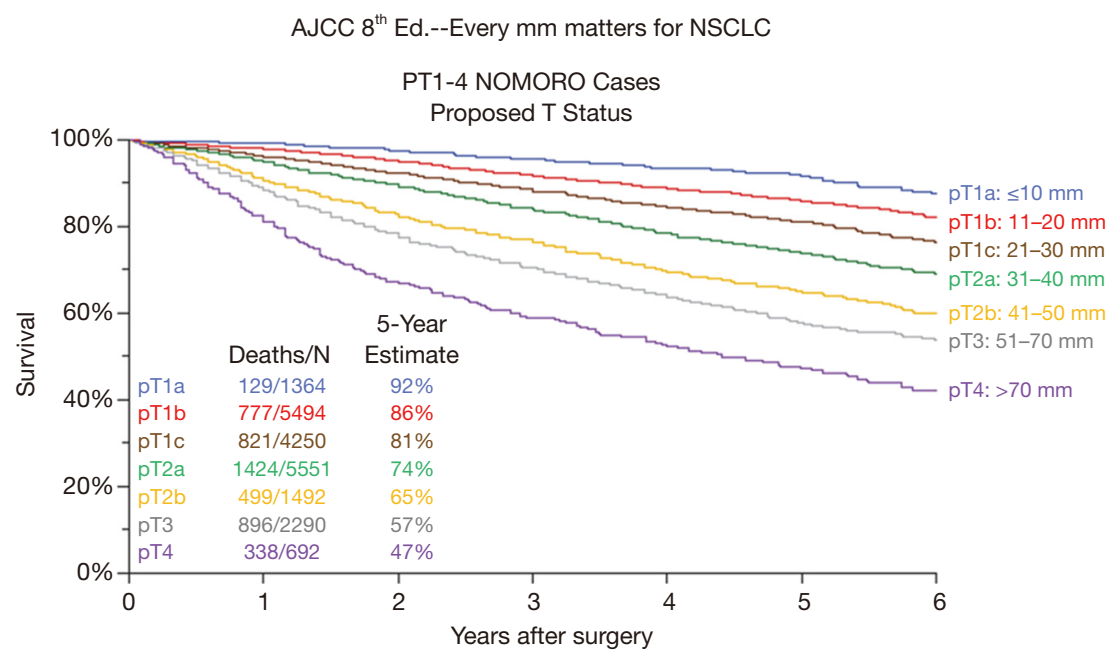

Figure 3 Delays from diagnosis to treatment reduces the benefits of early detection. The forthcoming $8^{\text {th }}$ edition of the American Joint Committee on Cancer (AJCC) staging system (51) has identified that the survival probability of NSCLC progressively declines with every millimeter of tumor growth that can result from delays in initiating treatment.

treatment (Figure 3).

Additional important factors that further increase the benefit of screening include a structured protocol for managing abnormal findings, quality oversight of LDCT interpretations, a dedicated navigator to ensure timely transition from diagnosis to treatment, access to a thoracic oncology multidisciplinary team, and continuous real-time feedback from a management system. These also account for the difference shown in Figure 1, between the I-ELCAP results and those in the NLST. The need for quality assurance of the imaging, interpretation, and follow-up was recognized as CMS requires statement of the protocol being used.

CMS also requires a SDM session between the person seeking screening and a physician or nurse practitioner before providing the LDCT. The SDM session should include the following topics related to LS risks and benefits. This session can take from 10 to 30 minutes.

Benefits: early detection and early treatment; incidental findings; experienced multidisciplinary team and established protocols; education about the screening process; teaching for future diagnostics; education on smoking cessation.

Risks: radiation exposure; false positive findings and possible complications; overdiagnosis; stress and anxiety; sharing personal information with relevant personnel within MSHS, although all privacy HIPAA privacy rules are followed; screening does not guarantee that you will be cured if a lung cancer is found.

At MSHS, this SDM discussion can be held by the referring physician guided by the EMR and both the person and referrer sign the form to certify that SDM has been completed. Alternatively, the physician can refer the person to the Mount Sinai LS program by the EMS, and the nurse practitioner can verify the eligibility, then perform the SDM session, and schedule the LDCT. The SDM verification form is signed by both the patient and nurse practitioner and is uploaded to the patient EMR.

At the PVAHCS, the vast majority of SDM sessions are conducted and documented by LS staff, whether the Veteran is contacted from the Precision Outreach pathway or whether the Veteran is referred by another clinician using the eConsult pathway. A few Veterans receive a LDCT for LS ordered by a clinician who may have not documented the SDM in the EMR. Once our LS staff is notified of the LDCT order by Radiology, the Veteran is interviewed and if the LS criteria and potential for medical benefit are verified, the LS staff conducts a SDM session and invites the Veteran to enroll in annual LS.

\section{Providing smoking cessation information and options}

Smoking cessation is a vital component of the screening program, not only for current smokers but also for former smokers to prevent relapse. CT screening provides a "teachable moment" for smoking cessation advice and has been shown to have no association with former smokers restarting the habit $(52,53)$. Personalized counseling or referral to "Quit Smoking Help Lines" and other support 
groups are useful in helping smokers quit or preventing relapse and such programs should be available. Smoking cessation advice is required by CMS.

At MSHS, smoking cessation counseling is provided by a nurse practitioner. The smoking cessation counseling session is performed in conjunction with the SDM discussion. The 5 A's of Tobacco Cessation Model is utilized to identify and address tobacco use: Ask, Advise, Assess, Assist, and Arrange. All current smokers are advised to quit smoking and informed of the benefits of smoking cessation. Assessment of patient's motivation and readiness to quit tobacco use are evaluated and documented based on a scale of 1 to 10. Barriers to quit smoking and reasons for tobacco use are also identified. Quit attempts, relapse triggers, coping strategies for urges, cravings and withdrawal symptoms are reviewed and addressed. Treatment options and recommendations regarding behavioral modification, nicotine replacement therapy and pharmacological methods are discussed, and prescribed. For those who have successfully quit using tobacco, challenges to remain quit and methods to prevent relapse are addressed. Importantly, the patient's commitment to a tobacco free lifestyle is reinforced. Smoking cessation packet is offered to all patients, which includes counseling support and referral information. Tobacco use and dependence are chronic relapsing conditions that, like other chronic diseases, may require repeated intervention and long-term support. The importance of continued smoking cessation counseling is reinforced for all current and former smokers.

At the PVAHCS, smoking cessation is also addressed during the SDM session, with education, resources and referrals to our smoking cessation program offered and made available when appropriate. There are numerous resources currently available at all VA medical centers that include written materials, face-to-face counseling, nicotine replacement, or medications through the VA national formulary. Veterans are informed about the 855-QUITVET (1-855-784-8838) quit line (http://vaww.publichealth. va.gov/smoking/quitline.asp), the SmokefreeVET textmessaging program (http://smokefree.gov/vet/), and a quit smoking mobile application. LS staff also informs Veterans that each VA Medical Center has a Smoking Cessation Lead Clinician who serves as the local clinical champion and the point of contact between each local medical center and national programs that can be reached at VHATobaccoProgram@va.gov (http://vaww.publichealth. va.gov/smoking/index.asp). Whether the Veteran was referred to the LS staff or contacted from the Precision
Outreach pathway, the LS note template documents in the EMR the SDM session and the communication about smoking cessation. The PCP or referring clinician is also copied in the LS staff note for general awareness or for prompting additional smoking cessation interventions.

\section{Obtaining the LDCT Screening and calibration of CT scanner and protocol performance}

Once the eligibility has been documented and information about the benefit and risks have been provided to the screening participant as well as the smoking cessation advice, the LDCT can be ordered. The LDCT imaging technique remains consistent between baseline and repeat screenings. Given that there are a large variety of CT manufacturers and scanner models with high-resolution capabilities, the following are general guidelines for image production. Scans should be acquired on MDCTs, ideally with scanners having more than 16 rows. Images should be acquired so that scans can be reconstructed with a slice thickness of $1 \mathrm{~mm}$ or less. Studies have shown that thinner slices are better for automated image processing and nodule detection.

There is no specific definition of "low-dose", although historically most screening protocols have used scan parameters of $120-140 \mathrm{kVp}$ and $30-100 \mathrm{mAs}$. I-ELCAP experience suggests that scans be obtained at $120 \mathrm{kVp}$ or lower and $40 \mathrm{mAs}$ (effective) or lower. Dose-modulation, collimation and pitch all affect the dose, and these should be set to allow for the lowest possible dose while maintaining acceptable image quality. Scan parameters should also be adjusted to allow for different size patients. Dose modulation techniques that adjust for body size are available on most modern scanners, or if not then scan parameters should be configured based on patient weight or BMI. Additionally, scan manufacturers are offering new dose reduction techniques, and their use is encouraged provided that acceptable image quality is maintained. Guidance on scan parameters specific to manufacturer make and model can be found on the website of the American Association of Physicists in Medicine (54) and by the RSNA QIBA guidelines $(41,42)$. Image reconstruction should be performed using a standard, non-edge enhancing kernel to minimize the effects of noise. However, additional reconstructions (e.g., sagittal, coronal) may also be obtained. Maximum intensity projection (MIP) images are useful for identifying nodules (55).

Images should be acquired in a single breath from the 
lung apices through the lung bases. Standards should be established to ensure consistent breath holding. It is important to note that contrast material is not used. Just prior to performing the low-dose CT scan, the participant is asked to cough vigorously several times to clear the trachea and major bronchi of possible mucus secretions, thereby avoiding additional imaging that might be required to distinguish such secretions from endobronchial lesions. Any follow-up imaging of abnormalities identified in screening studies should be performed using the same low dose parameters, without contrast material, that are used for the baseline and repeat screenings.

At MSHS, in addition to receiving ACR accreditation, the LDCT protocol performance was calibrated using a phantom developed for providing far more rigorous assessment of lung nodule measurements than the usual ACR CT accreditation phantom $(41,42)$.

At the PVAHCS, this phantom was also tested on the CT scanners used for LS, both at the main facility in Phoenix and at the NAVAHCS in Prescott, Arizona. On occasion a Veteran who wants to participate in LS may request that the LDCT be performed and a non-VA imaging center that is closer than the VA, and we are able to accommodate such requests. But it is not yet clear if we will be able to request those non-VA facilities to abide by the same calibration requirements that we have in the VA, or whether we may be able to assist those non-VA imaging centers that are serving our Veterans throughout the community to achieve the same rigorous performance standards.

\section{Interpretation of the LDCT screening results and providing follow-up recommendations}

The radiologist should be aware of the round (baseline or repeat) from which the images derives, as the work-up protocol depends on the specific screening round. The images are viewed on high-resolution monitors at their typical window and level settings-scrolling through the images one at a time. For the purposes of assessing the size of a nodule or that of a mediastinal abnormality, however, the following settings are typically used: lung window width 1,500 HU and lung window level -650 HU, and mediastinal window width $350 \mathrm{HU}$ and mediastinal window level $25 \mathrm{HU}$.

The LDCT findings should be reported using a structured format. This ensures that all relevant findings are reported in a consistent manner. The structured report facilities the communication with the referring physicians and provides all the relevant information for the radiologists reading the follow-up CTs and future annual repeat LDCTs. The recommendations should follow the specific protocol being used for the interpretations and recommendations. A lay report including the key findings and follow-up recommendations should be sent to the person who had the screening CT.

At MSHS, the LDCTs are read following the I-ELCAP protocol (24). It provides the definitions of the possible findings on the LDCT, the probability of malignancy and the follow-up recommendations. The structured format is displayed and the results of any previous LDCT are also displayed. The new findings are entered into the structured report which is then submitted as all radiology reports to the PACS system which distributes the report to the referring physicians, and it also places the report into our EMR "MYCHART" system which allows each person access to their own medical reports. The radiologist communicates any finding other than follow-up LDCT to the referring physician and discusses how best to communicate with the person who was screened.

At the PVAHCS, the LDCTs are read following the I-ELCAP protocol (24) and are also assigned a Lung-RADS score (56). When there have been minor discrepancies for reporting results and recommendations between I-ELCAP and Lung-RADS guidelines, such as rounding to the closest millimeter or recommending PET scan for screening, which are never recommended in I-ELCAP but have been sometimes considered in Lung-RADS, our multidisciplinary team of clinicians review the case and develop a consensus recommendation for follow up.

\section{Screening management system}

At MSHS, the ELCAP Management System ${ }^{\mathrm{TM}}$ is used to enter information on each person contacting the screening program. If the person wants to talk to the coordinator or schedule a LDCT, the system is used to schedule the time, including the SDM and smoking cessation sessions, and LDCT images, once obtained, are used to generate the structured report, and document it together with the follow-up recommendations. All subsequent screenings, diagnostic tests, and interventions related to the screening are entered. It provides the required CMS information on each radiology report and the transmission of the required data to a CMS-approved registry.

At the PVAHCS, the ELCAP Management System ${ }^{\mathrm{TM}}$ was installed on a dedicated Phoenix VA ELCAP server 
to be used for management of LS. The VA requires each medical contact with a Veteran to be documented in the VA Vista-CPRS EMR. This includes the initial contact with the Veteran, the discussion about the screening, the findings of the LDCT and the follow-up recommendations as well as any follow-up contact for further workup or for the next annual LDCT screening. For this reason, the ELCAP Management System ${ }^{\mathrm{TM}}$ program code and forms were licensed to develop the "VA-ELCAP" version of the ELCAP Management System ${ }^{\mathrm{TM}}$. Some forms have already been used to create Vista-CPRS EMR templates, enabling capturing of data that is integrated with the Phoenix ELCAP Dashboard for precision outreach of Veterans enrolled in LS. The goal for the future state is to have the "VA-ELCAP" version of the ELCAP Management System ${ }^{\text {TM }}$ fully translated into MUMPS and when functional, fully integrated with the VA VistaCPRS EMR (22).

\section{Quality assurance, feedback, and outcomes analyses}

Quality assurance, feedback, and analyses of outcomes are critical components to achieve the full benefit of LS. This includes training of all involved in the screening in all aspects of LS, including all the LS nurses, navigators, and physicians who talk to the person seeking the screening, those interpreting the LDCT screening, and who provide follow-up and, if diagnosed with lung cancer, those who provide the treatment and post-treatment care.

The centralized process from a comprehensive management system provides access for QA reviews by expert chest radiologists to ensure appropriate image acquisition, interpretation, and coding. The reviews provide discrepancy reports with conference calls to discuss the summary findings. This process was used for I-ELCAP institutions joining I-ELCAP. We found that new LS programs are more likely to report positive finding, most likely because of the concern of missing early lung cancers. A comparison of the first 100 LS interpretations compared to the dual reading by the I-ELCAP center radiologists with subsequent follow-up discussions, and successive evaluations showed convergence in the interpretations (31). Similarly, a QA system of the interpretations and followup recommendations will be developed at the PVAHCS through a similar, but inward-facing system. This latter effort, in collaboration with the VA National Teleradiology Program, will aim to explore the potential for expansion of this service to any VA medical center in the country that is offering LS services, regardless of their participation in this project.

The QA initiatives include:

* LDCT dual reads: the PVAHCS QA team (e.g., CH, DY) will dual-read the first 100 cases reported by each VA radiologist at the other VA sites, and make sitevisits to provide feedback. This process will become scalable by having additional radiologists join in this process so that additional sites can be evaluated.

* Protocol compliance: software programs will evaluate all scans at each site to confirm LS eligibility criteria were met, and to ensure follow up recommendations are being followed.

* Biopsy alerts: CT forms that recommend a biopsy automatically alert the QA team at PVAHCS for rapid review and feedback to sites. This will be expanded to include other radiologists to become involved in the evaluation.

* Scanner quality checks: maintain a summary of phantom results for each scanner in use, and provide support for sites to optimize image capture.

- Continuous scanner QA: develop an automatic process that confirms image quality for every LDCT. This will include software programs that evaluate the actual CT scan images and provide feedback regarding quality. This includes a variety of features including radiation dose, appropriate scan length, noise assessment. In addition, as new technology is developed for monitoring the scanners themselves, it will be deployed.

Provide a monthly summary report for each site detailing the frequency of the largest nodule and the recommendations for further workup, and the resulting diagnoses by stage of lung cancer.

Training. The implementation of the ELCAP Management System ${ }^{\text {TM }}$ for the I-ELCAP sites throughout the world did not merely push out a set of guidelines to follow with a set of tools. Instead, it embraced close communication from the start to facilitate accurate understanding of the protocol and identifying how to optimize the benefits of screening (31). When followed appropriately, it ensured minimization of risks of erroneous interpretations that lead to unnecessary invasive procedures for benign nodules that are not growing. It also assured accurate identification of suspicious nodules and other radiographic abnormalities in the chest that warrant further workup during any round of screening.

Radiologists: Each radiologist will be trained in the 
screening protocol, and how to communicate the results to people being screened. Each will have individualized training with ongoing continuous education through written materials, electronic teaching files, and site visits to provide support from a team of experts, whenever and wherever the need may arise. Formalized training will focus on the importance of adherence to the I-ELCAP guidelines for managing findings in both the initial baseline as well as annual repeat round of screening.

The structured reporting allows for characterization of the nodule findings as well as other findings. This allows for substantial collection of high-quality clinical data to facilitate a rigorous evaluation of the population being screened.

(I) Radiology teaching files: a repository of cases was developed to train radiologists on interpretation of LDCT scans, whether reporting with LungRADS or I-ELCAP. Cases are presented with answers and suggestions on accompanying slides, and cover a broad range of categories that relate to findings both common and uncommon in the screening setting (minimum of 100 cases).

(II) Radiology certification program: a series of test cases will evaluate radiologists' skills with response assessments with a view towards assessing proper use of standardized reporting systems. Quizzes will be scored to facilitate one-on-one feedback.

A radiology quality assurance program will be introduced. This is considered an important contribution to the success of any large LS program given the potential for variability that can lead to unnecessary and sometimes harmful invasive procedures.

Navigators. Each navigator will be trained on how to identify, counsel, and safely track patients at risk for lung cancer. Their primary role will be to counsel patients interested in screening, communicate abnormal findings with local clinicians to facilitate appropriate and timely follow-up. Perhaps most importantly, they will also help ensure timely transition from diagnosis to treatment, given delays to care are known to compromise survival and the opportunity for cure.

Standardized training protocols will be used to train navigators with teleconferences, site visits, and workshops to ensure navigators are aware about the nuances of the patient management system. There will be an emphasis on learning how to lead a shared discussion. To decreases some patient's propensity to smoke, navigators will also be taught to take advantage of the "teachable moment" that screening offers to counsel patients about smoking cessation. This will include former, as well as current, smokers given their potential for relapse. This provides not only an additional effective measure to reduce the risk of death from lung cancer, but also improve Veterans' cardiovascular health which provides an even larger benefit than the early detection of lung cancer and can occur even more quickly.

As the original ELCAP Management System ${ }^{\mathrm{TM}}$ is installed and further improved at these two health care organizations, these maturing systems will automate many of the clinical processes that are needed to successfully monitor large cohorts of patients undergoing LS. A key advantage is that it will reduce the need for manual data entry, reduce the opportunity for unintentional entry errors, automate alerts for clinicians whenever patients are overdue for their next evaluation(s), and develop a structured dataset of clinical information that will be later used for quality assurance and program evaluation.

\section{Conclusions}

Lung screening has the potential to drastically reduce the number of lung cancer deaths, but to maximize the benefits it needs to be implemented with care. There are many different facets to consider, not only the actual process of obtaining the CT scan, interpreting the results, and recommending follow-up, but also the challenges of the workflow, eligibility, participation of primary care physicians and specialists, just to name a few. We have provided two examples of well-implemented lung screening programs, one in a large private hospital system and the other in a busy VA health care system, describing how many of these challenges can be overcome to provide lung screening to large and diverse populations. The critical components are the LS staff and a comprehensive management system.

\section{Acknowledgments}

The authors wish to dedicate this publication to the memory of our dear colleague and friend, Dr. Danny Kilpatrick, former Chief of Radiology at the PVAHCS and a relentless supporter of lung screening for Veterans. The views presented in this manuscript are those of the authors and do not reflect the views of the U.S. Government or the Department of Veterans Affairs. The PVAHCS work was supported by the PVAHCS, the VA Office of Rural Health, and the Bristol Myers Squibb Foundation. The Phoenix ELCAP Dashboard is work product from 
the PVAHCS Clinical Informatics Service, Hamed Abbaszadegan, MD, Chief Health Informatics Officer. For additional information on SQL code and implementation of the Phoenix ELCAP Dashboard and related clinical notes templates, please contact Peter A. Fredricks (Peter. Fredricks@va.gov) and Daniel P. Garcia (Daniel.Garcia7@ va.gov). For additional information about clinical processes and policies related to the use of the Phoenix ELCAP Dashboard and related clinical templates, please contact Providencia Morales (Providencia.Morales@va.gov). MSHS work has been supported by the Simons Foundation and Bristol Myers Squibb Foundation.

Funding: None.

\section{Footnote}

Provenance and Peer Review: This article was commissioned by the Guest Editor (Witold Rzyman) for the series "Implementation of CT-based screening of lung cancer" published in Translational Lung Cancer Research. The article has undergone external peer review.

Conflicts of Interest: All authors have completed the ICMJE uniform disclosure form (available at http://dx.doi. org/10.21037/tlcr-20-761). The series "Implementation of CT-based screening of lung cancer" was commissioned by the editorial office without any funding or sponsorship. $\mathrm{CIH}$ is a named inventor on a number of patents and patent applications relating to the evaluation of pulmonary nodules on CT scans of the chest which are owned by Cornell Research Foundation (CRF). Since 2009, CIH does not accept any financial benefit from these patents including royalties and any other proceeds related to the patents or patent applications owned by CRF. CIH is the President and serve on the board of the Early Diagnosis and Treatment Research Foundation. She receives no compensation from the Foundation. The Foundation is established to provide grants for projects, conferences, and public databases for research on early diagnosis and treatment of diseases. Recipients include, I-ELCAP, among others. The funding comes from a variety of sources including philanthropic donations, grants and contracts with agencies (federal and non-federal), imaging and pharmaceutical companies relating to image processing assessments. The various sources of funding exclude any funding from tobacco companies or tobacco-related sources. DFY reports other from Accumetra, other from GRAIL, outside the submitted work; In addition, DFY is a named inventor on a number of patents and patent applications relating to the evaluation of diseases of the chest including measurement of nodules. Some of these, which are owned by Cornell Research Foundation (CRF), are non-exclusively licensed to General Electric. As an inventor of these patents, he is entitled to a share of any compensation which CRF may receive from its commercialization of these patents. He is also an equity owner in Accumetra, a privately held technology company committed to improving the science and practice of imagebased decision making. DFY also serves on the advisory board of GRAIL. The authors have no other conflicts of interest to declare.

Ethical Statement: The authors are accountable for all aspects of the work in ensuring that questions related to the accuracy or integrity of any part of the work are appropriately investigated and resolved.

Open Access Statement: This is an Open Access article distributed in accordance with the Creative Commons Attribution-NonCommercial-NoDerivs 4.0 International License (CC BY-NC-ND 4.0), which permits the noncommercial replication and distribution of the article with the strict proviso that no changes or edits are made and the original work is properly cited (including links to both the formal publication through the relevant DOI and the license). See: https://creativecommons.org/licenses/by-nc-nd/4.0/.

\section{References}

1. Henschke CI, Miettinen O, Yankelevitz D, et al. Radiographic screening for cancer. Proposed paradigm for requisite research. Clin Imaging 1994;18:16-20.

2. Henschke CI, McCauley D, Yankelevitz D, et al. Early Lung Cancer Action Project: overall design and findings from baseline screening. Lancet 1999;354:99-105.

3. Henschke CI, Naidich D, Yankelevitz D, et al. Early lung cancer action project: initial findings on repeat screenings. Cancer 2001;92:153-9.

4. International Early Lung Cancer Action Program Investigators, Henschke CI, Yankelevitz DF, et al. Survival of patients with stage I lung cancer detected on CT screening. N Engl J Med 2006;355:1763-71.

5. Henschke CI, Wisnivesky JP, Yankelevitz DF, et al. Small stage I cancers of the lung: genuineness and curability. Lung Cancer 2003;39:327-30. 
6. Henschke CI, Yankelevitz DF, Miettinen OS. Computed tomographic screening for lung cancer: the relationship of disease stage to tumor size. Arch Intern Med 2006;166:321-5.

7. Mulshine JL. One Screening for Ischemic Heart Disease, Lung Cancer, and Chronic Obstructive Pulmonary Disease: A Systems Biology Bridge for Tobacco and Radiation Exposure. Am J Public Health 2018;108:1294-5.

8. Sone S, Nakayama T, Honda T, et al. Long-term followup study of a population-based 1996-1998 mass screening programme for lung cancer using mobile low-dose spiral computed tomography. Lung Cancer 2007;58:329-41.

9. Aberle DR, Adams A, Berg C, et al. Reduced lung-cancer mortality with low-dose computed tomographic screening. N Engl J Med 2011;365:395-409.

10. de Koning HJ, van der Aalst CM, de Jong PA, et al. Reduced Lung-Cancer Mortality with Volume CT Screening in a Randomized Trial. N Engl J Med 2020;382:503-13.

11. Moyer VA, U. S. Preventive Services Task Force. Screening for lung cancer: U.S. Preventive Services Task Force recommendation statement. Ann Intern Med 2014;160:330-8.

12. Proposed Decision Memo for Screening for Lung Cancer with Low Dose Computed Tomography (LDCT) (CAG00439N). Centers for Medicare and Medicaid Services (CMS), Washington, DC. Available online: http:// www.cms.gov/medicare-coverage-database/details/ncaproposed-decision-memo.aspx? NCAId=274

13. American Cancer Society. Cancer Facts \& Figures 2020. Atlanta, GI: American Cancer Society 2020.

14. Yang P, Wang Y, Wampfler JA, et al. Trends in Subpopulations at High Risk for Lung Cancer. J Thorac Oncol 2016;11:194-202.

15. Wang Y, Midthun DE, Wampfler JA, et al. Trends in the proportion of patients with lung cancer meeting screening criteria. JAMA 2015;313:853-5.

16. Aldrich MC, Mercaldo SF, Sandler KL, et al. Evaluation of USPSTF Lung Cancer Screening Guidelines Among African American Adult Smokers. JAMA Oncol 2019;5:1318-24.

17. Doria-Rose VP, White MC, Klabunde CN, et al. Use of Lung Cancer Screening Tests in the United States: Results from the 2010 National Health Interview Survey. Cancer Epidemiol Biomarkers Prev 2012;21:1049-59.

18. Liu B, Dharmarajan K, Henschke CI, et al. State-level variations in the utilization of lung cancer screening among fee-for-service Medicare beneficiaries: an analysis of the 2015-2017 Physician and Other Supplier data. Chest 2020;157:1012-20.

19. Jemal A, Fedewa SA. Lung Cancer Screening With LowDose Computed Tomography in the United States-2010 to 2015. JAMA Oncol 2017;3:1278-81.

20. Richards TB, Doria-Rose VP, Soman A, et al. Lung Cancer Screening Inconsistent With US Preventive Services Task Force Recommendations. Am J Prev Med 2019;56:66-73.

21. Lung cancer cohort data from VA VINCI Dataset, PVAHCS IRB Approved Project, Title: "Evaluation of Lung Cancer Screening Criteria That May Have Saved More Veterans' Lives", Investigators: Samuel M. Aguayo, Paska A. Permana, Janet Fawcett, Samir Sultan.

22. Henschke C. An Open Source Lung Screening Management System. Presented at the IASLC 2019 WCLC World Conference on Lung Cancer.; October: Journal of Thoracic Oncology; 2019. p. S220-1.

23. NY-ELCAP Investigators. CT Screening for lung cancer: diagnoses resulting from the New York Early Lung Cancer Action Project. Radiology 2007;243:239-49.

24. International Early Lung Cancer Action Program Investigators. International Early Lung Cancer Action Program protocol. www.IELCAP.org/protocols. Accessed Jan 3 rd 2018.

25. Hays MT. A historical look at the establishment of the Department of Veterans Affairs Research \& Development Program. Veterans Health Administration, Research, Development; 2010.

26. International Early Lung Cancer Action Program Investigators. Program and Consensus statements. International Conferences on Screening for Lung Cancer. Available online: http://events.ielcap.org/conferences/past. Accessed September 42019.

27. Kinsinger LS, Anderson C, Kim J, et al. Implementation of Lung Cancer Screening in the Veterans Health Administration. JAMA Intern Med 2017;177:399-406.

28. Henschke CI, Yip R, Yankelevitz DF, et al. Definition of a positive test result in computed tomography screening for lung cancer: a cohort study. Ann Intern Med 2013;158:246-52.

29. Yip R, Henschke CI, Yankelevitz DF, et al. CT Screening for Lung Cancer: Alternative Definitions of Positive Test Result Based on the National Lung Screening Trial and International Early Lung Cancer Action Program Databases. Radiology 2014;273:591-6. 
30. Kazerooni EA, Armstrong MR, Amorosa JK, et al. ACR CT accreditation program and the lung cancer screening program designation. J Am Coll Radiol 2015;12:38-42.

31. Xu DM, Lee IJ, Zhao SJ, et al. CT Screening for Lung Cancer: Value of Expert Review of Initial Baseline Screenings. AJR Am J Roentgenol 2015;204:281-6.

32. Reeves A, Kostis W, Yankelevitz D, et al. A Web-based database system for multi-institutional research studies on lung cancer. Radiology 2001;221:372.

33. Serving Those Who Served: Bristol-Myers Squibb Foundation has teamed up with the U.S. Department of Veterans Affairs to help improve lung cancer survival rates for veterans. December 4, 2017. Bristol-Myers Squibb Foundation. 2017. Available online: https://www.bms.com/ about-us/responsibility/bristol-myers-squibb-foundation/ our-success-stories/serving-those-who-served.html. Accessed September 42019.

34. Wood DE, Kazerooni EA, Baum SL, et al. Lung Cancer Screening, Version 3.2018. J Natl Compr Canc Netw 2018;16:412-41.

35. Krefft SD, Rose CS, Nawaz S, et al. Deployment-Related Lung Disorders. Federal practitioner: for the health care professionals of the VA, DoD, and PHS 2015;32:32-8.

36. International Early Lung Cancer Action Program I, Henschke CI, Yip R, et al. Women's susceptibility to tobacco carcinogens and survival after diagnosis of lung cancer. JAMA 2006;296:180-4.

37. Mannino DM, Aguayo SM, Petty TL, et al. Low lung function and incident lung cancer in the United States: data From the First National Health and Nutrition Examination Survey follow-up. Arch Intern Med 2003;163:1475-80.

38. Field JK, Smith RA, Aberle DR, et al. International Association for the Study of Lung Cancer Computed Tomography Screening Workshop 2011 report. J Thorac Oncol 2012;7:10-9.

39. Kauczor HU, Bonomo L, Gaga M, et al. ESR/ERS white paper on lung cancer screening. Eur Radiol 2015;25:251931.

40. Yip R, Henschke C, Yankelevitz D, et al. The impact of the regimen of screening on lung cancer cure: a comparison of I-ELCAP and NLST. Eur J Cancer Prev 2015;24:201-8.

41. Quantitative Imaging Biomarkers Alliance (QIBA). QIBA Profile: Small Lung Nodule Volume Assessment and Monitoring in Low Dose CT Screening. Radiological Society of North America. 2018. Available online: http:// qibawiki.rsna.org/images/a/a8/QIBA_CT_Vol_SmallLung
NoduleAssessmentInCTScreening_2018.11.18-clean.pdf. Accessed September 42019.

42. Radiological Society of North America Quantitative Imaging Biomarkers Alliance (QIBA) Nodule Profile Calculator. 2017. Available online: https://accumetra.com/ qiba-nodule-profile-calculator/. Accessed September 4 2019.

43. American College of Radiology (ACR). Lung CT screening reporting \& data system (Lung-RADS Version 1.0). 2014. Available online: https://www.acr.org/-/ media/ACR/Files/RADS/Lung-RADS/LungRADS_ AssessmentCategories.pdf?la=en. Accessed September 11 2019.

44. Henschke CI, Yip R, Ma T, et al. CT screening for lung cancer: comparison of three baseline screening protocols. Eur Radiol 2019;29:5217-26.

45. Henschke CI, Salvatore M, Cham M, et al. Baseline and annual repeat rounds of screening: implications for optimal regimens of screening. Eur Radiol 2018;28:1085-94.

46. Henschke CI, Yip R, Shaham D, et al. The Regimen of Computed Tomography Screening for Lung Cancer. Lessons Learned Over 25 Years From the International Early Lung Cancer Action Program. J Thorac Imaging 2021;36:6-23.

47. Libby DM, Wu N, Lee IJ, et al. CT screening for lung cancer: the value of short-term CT follow-up. Chest 2006;129:1039-42.

48. Wisnivesky JP, Yankelevitz D, Henschke CI. The effect of tumor size on curability of stage I non-small cell lung cancers. Chest 2004;126:761-5.

49. Yankelevitz D, Wisnivesky JP, Henschke CI. Stage of lung cancer in relation to its size: part 1 . Insights. Chest 2005;127:1132-5.

50. Wisnivesky JP, Yankelevitz D, Henschke CI. Stage of lung cancer in relation to its size: part 2. Evidence. Chest 2005;127:1136-9.

51. Rami-Porta R, Bolejack V, Crowley J, et al. The IASLC Lung Cancer Staging Project: Proposals for the Revisions of the T Descriptors in the Forthcoming Eighth Edition of the TNM Classification for Lung Cancer. J Thorac Oncol 2015;10:990-1003.

52. Ostroff JS, Buckshee N, Mancuso C, et al. Smoking cessation following CT screening for early detection of lung cancer. Prev Med 2001;33:613-21.

53. Anderson CM, Yip R, Henschke CI, et al. Smoking cessation and relapse during a lung cancer screening program. Cancer Epidemiol Biomarkers Prev 
2009;18:3476-83.

54. American Association of Physicists in Medicine. Hazards Of CT Scans Overstated, According To Physicist.

ScienceDaily. December 31, 2007. www.sciencedaily.com/ releases/2007/11/071130173233.htm.

55. Liang M, Yip R, Tang W, et al. Variation in Screening CTDetected Nodule Volumetry as a Function of Size. AJR
Am J Roentgenol 2017;209:304-8.

56. American College of Radiology (ACR). Lung-RADS v1.1 Assessment Categories. 2019. Available online: https:// www.acr.org/-/media/ACR/Files/RADS/Lung-RADS/Lu ngRADSAssessmentCategoriesv1-1.pdf?la=en. Accessed September 112019.

Cite this article as: Henschke CI, Yankelevitz DF, Jirapatnakul A, Yip R, Reccoppa V, Benjamin C, Llamo T, Williams A, Liu S, Max D, Aguayo SM, Morales P, Igel BJ, Abbaszadegan H, Fredricks PA, Garcia DP, Permana PA, Fawcett J, Sultan S, Murphy LA. Implementation of low-dose CT screening in two different health care systems: Mount Sinai Healthcare System and Phoenix VA Health Care System. Transl Lung Cancer Res 2021;10(2):1064-1082. doi: 10.21037/tlcr-20-761 